Research Article

\title{
Chitosan Nanoparticles to Enhance the Inhibitory Effect of Natamycin on Candida albicans
}

\author{
Yaling Liu $\mathbb{D}^{1},{ }^{1}$ Xiaoming Cui $\mathbb{D},{ }^{2}$ Liping Zhao $\mathbb{D}{ }^{2}$ Weifen Zhang $\mathbb{D},,^{2,3,4}$ Shourong Zhu $\mathbb{D}^{1}$, \\ and Jinlong $\mathrm{Ma}$ iD $2,3,4$ \\ ${ }^{1}$ Department of Ophthalmology, Affiliated Hospital of Weifang Medical University, Weifang, 261031 Shandong, China \\ ${ }^{2}$ College of Pharmacy, Weifang Medical University, 7116\# Baotong West Street, Weifang, 261053 Shandong, China \\ ${ }^{3}$ Collaborative Innovation Center for Target Drug Delivery System, Weifang Medical University, Weifang, 261053 Shandong, China \\ ${ }^{4}$ Shandong Engineering Research Center for Smart Materials and Regenerative Medicine, Weifang Medical University, Weifang, \\ 261053 Shandong, China
}

Correspondence should be addressed to Shourong Zhu; yknet@sina.com and Jinlong Ma; majinlong99@hotmail.com

Received 27 October 2020; Revised 11 March 2021; Accepted 13 March 2021; Published 5 April 2021

Academic Editor: Antonio Vassallo

Copyright (C) 2021 Yaling Liu et al. This is an open access article distributed under the Creative Commons Attribution License, which permits unrestricted use, distribution, and reproduction in any medium, provided the original work is properly cited.

Fungal keratitis is a stubborn fungal infection that is widespread worldwide. It can even affect the health and life of a patient. At present, natamycin (NAT) is the first-line drug in the treatment of fungal keratitis, despite its disadvantages of clinical use, such as low drug bioavailability and poor water solubility. Herein, inspired by the adhesion properties of chitosan and its excellent drug loading and antifungal properties, we designed simple natamycin-chitosan nanoparticles (NAT-NPs) to investigate the feasibility of chitosan with NAT for eye treatment. Results showed that the NAT-NPs increased the antifungal effect of NAT due to the antifungal feature of chitosan NPs. Therefore, NAT-NPs are expected to become potential candidates for the treatment of fungal keratitis due to their high bacteriostasis.

\section{Introduction}

In recent years, the incidence of fungal keratitis has increased significantly due to the rise in vegetative corneal trauma, improper use of contact lenses, and widespread use of antibiotics and hormones [1-4]. Fungal keratitis typically occurs in autumn and affects mostly males and middle-aged and elderly farmers; most patients have a history of trauma and local medication. Fungal keratitis results in severe visual impairment, and its treatment is more difficult than other corneal infections $[5,6]$. Although the gold standard for the treatment of fungal keratitis has not been identified $[7,8]$, experts believe that natamycin (NAT) is a tetraene polyene that is the most important agent in the management of fungal keratitis [9]. Its mechanism of action combines the hydro- phobic part of the molecule with the ergosterol of the fungal cell membrane to form a polyene steroid complex through Van der Waal forces, thereby changing the permeability of the cell membrane. It then causes the failure of ions, nucleotides, and amino acids in fungal cells, disrupting the normal metabolism of cells and inhibiting fungi [10]. The current NAT formulation and dosage regimen consist of $5 \%(w / v)$ ophthalmic suspension instilled in the conjunctival sac at hourly or two-hourly intervals for several days. However, frequent dosing is required with high drug loading (DL) to maintain the drug levels within the therapeutic window, which can result in poor compliance in patients $[11,12]$.

The cell in the cornea is relatively special and features a lipid-water-lipid sandwich-layered structure. The poor water solubility of NAT hinders its penetration into deep corneal 
layers and the anterior chamber, which limits its use as monotherapy only for superficial keratitis $[13,14]$. Moreover, the drug is removed from the ocular surface within a few minutes due to the rapid clearance of drug by fast tear turnover, blinking, and lacrimal drainage; these events are the main reason for the low drug bioavailability of less than $5 \%$ [15-18]. To improve the residence time in front of the eyes, chitosan (CTS), poly(acrylic acid) acid, alginic acid, pectin, hyaluronic acid, and lecithin have been used as adhesive materials; these compounds have shown promising results [19-26]. Ramish et al. developed 5-fluorouracil-loaded CTS-coated sodium alginate-CTS nanoparticles (NPs) for ophthalmic administration. The retention time in the anterior cornea area was prolonged, improving bioavailability [24]. Therefore, drugs may be carried with adhesive materials for solubility and adhesion time, which in turn increases drug bioavailability.

CTS is a polycationic biopolymer with unique physicalchemical (e.g., thermal stability and $\mathrm{pH}$ sensitivity), biological (e.g., antimicrobial activity), and mechanical (e.g., film forming) properties [27]. The nontoxic nature of the polymer permits its use for biomedical applications, while its antimicrobial, antifungal, and wound healing features lead to research of further possible uses [28, 29]. The positive charge that the CTS shell carries can bind to the negatively charged corneal surface to increase the residence time of a drug in front of the cornea [30]. In addition, CTS has good inhibitory activity against Escherichia coli, Staphylococcus aureus, and Candida albicans [31]. For example, Bhatta et al. developed natamycin-embedded lecithin-CTS adhesion NPs by CTS crosslinking [32]; the bioavailability of the drug on the eye was found to significantly increase, facilitating its penetration into the inner layers. However, the enhanced antifungal effect of CTS on NAT is unclear; so, further investigations without liposomes are needed.

In this article, CTS and TPP were used as wrappers to wrap NAT into NAT-NPs to enhance the inhibitory effect of NAT on C. albicans (Figure 1). These NAT-NPs were characterized by their mean particle size $=369.2 \pm 6.65 \mathrm{~nm}$, polydispersity index $(\mathrm{PDI})=0.280$, encapsulation efficiency $(\mathrm{EE})=92.94 \% \pm 0.21 \%$, theoretical DL $=29.40 \% \pm 1.34 \%$, and zeta potential $=35.5 \pm 2.45 \mathrm{mV}$. In vitro release exhibited sustained release of $62.7 .0 \% \pm 0.14 \%$ for $12 \mathrm{~h}$ with an initial burst release of $31.7 \% \pm 0.70 \%$ in the first hour. The IC50 and zone of inhibition of NAT-NPs showed stronger antifungal activity than NAT against C. albicans. We also found that the antifungal feature of CTS-NPs made a remarkable contribution to the excellent antifungal effect of NAT-NPs. Thus, sodium tripolyphosphate/CTS-NPs are expected to become potential candidates for the treatment of fungal keratitis due to their high bacteriostasis.

\section{Materials and Methods}

2.1. Materials. CTS (DAC $=96.1 \%$, molecular weight $=12 \mathrm{kD}$ ) was obtained from Hai Debei Marine Biotechnology Company (Jinan, People's Republic of China). TPP $\left(\mathrm{P}_{2} \mathrm{O}_{5}\right.$ content $\left.=58 \%\right)$ was purchased from Yantai Far Eastern Fine Chemical Co., Ltd. (Shandong, People's Republic of China). Dimethyl sulfoxide (DMSO; content $\geq 99.5 \%$ ) was purchased from Damao Chemical Reagent Factory. Pimaricin (NAT; BR $=95 \%$ ) was purchased from Shanghai Yuanye Bio-Technology Co., Ltd. (Shanghai, People's Republic of China). Tween-80 was purchased from Hengxing Reagents Co., Ltd. (Tianjin, People's Republic of China).

Liquid Sabouraud medium and Sabouraud agar were purchased from Hopebio Qingdao Hope Bio-Technology Co., Ltd. (Qingdao, People's Republic of China). TTC was purchased from BBI Life Sciences. C. albicans (Robin Berkhout ATCC ${ }^{\circledR}$ 18804) was purchased from Credible Leads to Incredible. A digital display vernier caliper was purchased from Senda Tools Co., Ltd. (Zhejiang, People's Republic of China).

2.2. Preparation of NAT-NPs. NAT-loaded NPs were prepared by ionic gelation [33]. The fabrication of NAT-NPs is illustrated in Figure 1. First, we dissolved NAT in DMSO under ultrasound conditions in a warm water bath to prepare a $1 \mathrm{mg} / \mathrm{mL}$ NAT solution. CTS was solubilized in aqueous $1 \%$ $(\mathrm{v} / \mathrm{v})$ acetic acid at a concentration of $1 \%(\mathrm{w} / \mathrm{v})$. TPP was solubilized in deionized water at a concentration of $1 \%(\mathrm{w} / \mathrm{v})$. The NAT-NPs suspensions were obtained by injecting water-diluted TPP solutions into the mixture with different volumes of NAT solution injected into water-diluted CTS solutions. All processes were carried out under magnetic stirring at $500 \mathrm{rpm}$. The volume ratio of CTS to TPP was $5: 1$, whereas the volume ratios of NAT to CTS and TPP were $1: 20,1: 12,2: 15,1: 6,1: 4$, and $1: 3$. Blank NPs (without drug) were prepared following the same procedure. The prepared NPs were preserved after dialysis. The NAT-NPs suspensions were dialyzed against deionized water at room temperature for $48 \mathrm{~h}$ using a membrane with an MWCO of 3500 Da (Spectrum Laboratories Inc., USA).

2.3. Particle Size and PDI. The size distribution and zeta potential of NPs were determined by photon correlation spectroscopy with a Zetasizer Nano ZS Zen 3600 (Malvern Instruments, Inc.). Size distribution analysis and PDI were performed at $25^{\circ} \mathrm{C}$. The zeta potential was measured by electrophoretic light scattering using a disposable zeta cuvette. All measurements were performed in triplicate, and results are presented as mean $\pm \mathrm{SD}$.

2.4. Morphological Characteristics by Transmission Electron Microscopy (TEM). The structure and topography of the NAT-NPs were examined by TEM analysis (CM30 electron microscope; Philips, Amsterdam, the Netherlands) at an acceleration voltage of $100 \mathrm{kV}$. One drop of the sample dispersion was carefully casted onto a clean copper grid. Extra solution was air-dried and directly observed under TEM without staining.

2.5. DL and EE. A measured amount of NAT-NPs formulation $(6.5 \mathrm{~mL})$ was centrifuged at a high speed $(12,000 \mathrm{rpm}$ for $30 \mathrm{~min})$. Subsequently, the supernatant was collected and analyzed to determine the free NAT content, and the sediment was dried and weighed to estimate the DL and EE of NAT in NAT-NPs. The NAT content was determined by using a UV-visible light spectrophotometer (UV) at 


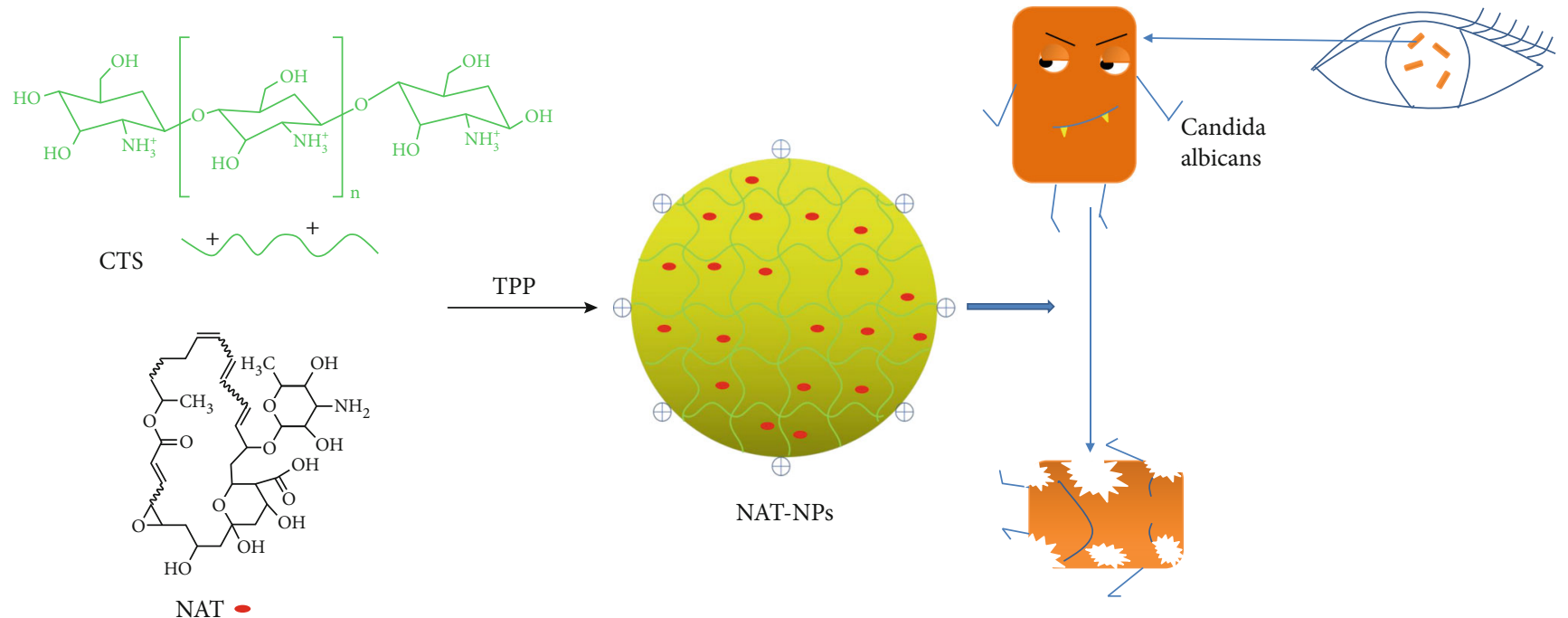

Figure 1: Preparation process of the NAT-NPs and the chemical structures of the components.
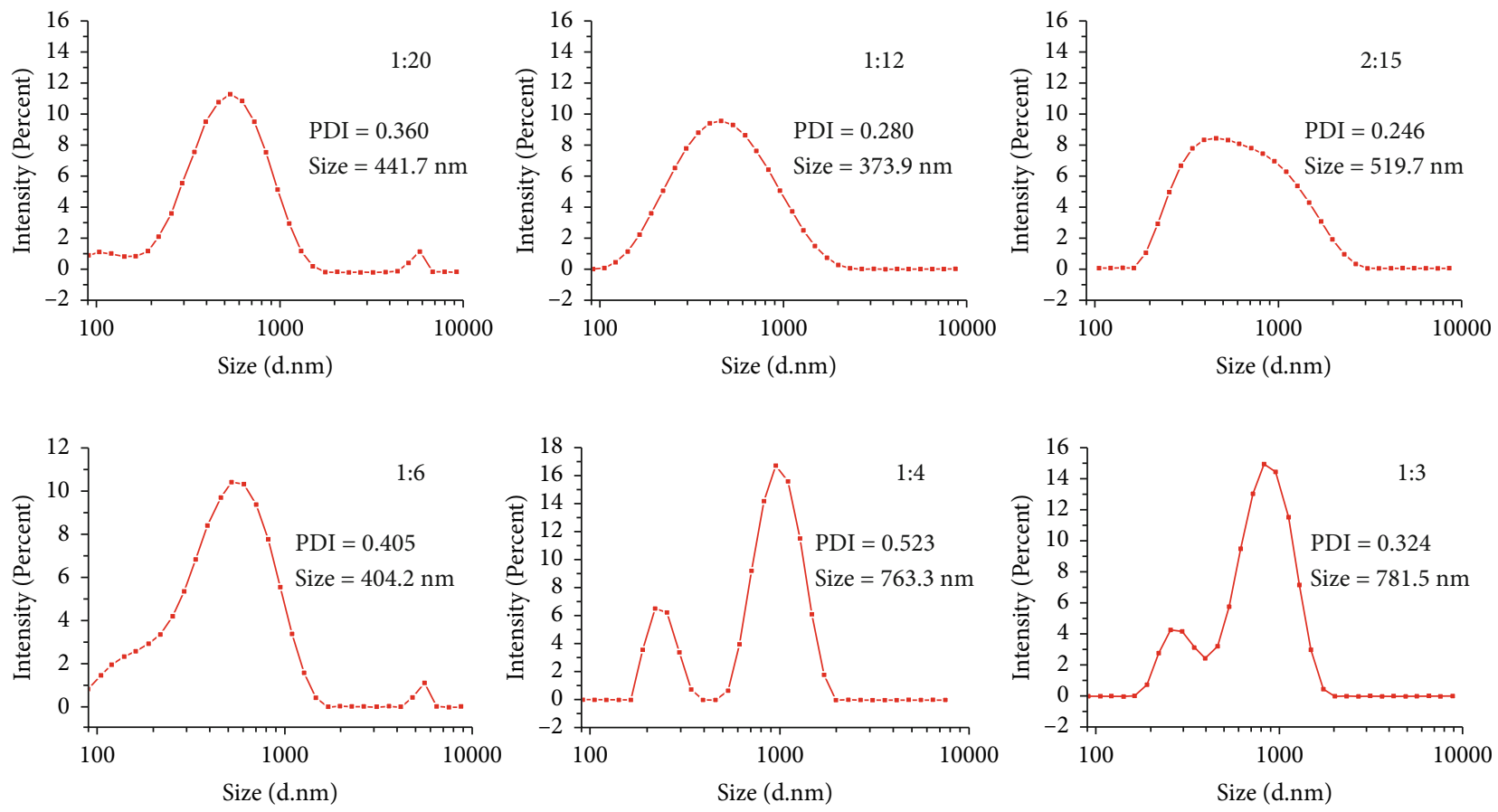

Figure 2: The size distribution of different volume ratio of NAT to carriers.

$303 \mathrm{~nm}$, and a standard curve was obtained for NAT. DL (\%) and $\mathrm{EE}(\%)$ were calculated from the following equations:

$$
\begin{gathered}
\mathrm{DL}(\%)=\frac{\text { actual amount of drug encapsulated in NPs }}{\text { amount of NPs }} \times 100 \%, \\
\mathrm{EE}(\%)=\frac{\text { actual amount of drug encapsulated in NPs }}{\text { initial amount of drug used in the fabrication of NPs }} \times 100 \% .
\end{gathered}
$$

2.6. FTIR and TGA Analyses. FT-IR analysis was conducted using an FT-IR Avater-360 spectrometer (PerkinElmer,
Waltham, MA, USA), which scanned in the region of $4,000-400 \mathrm{~cm}^{-1}$ for each spectrum at a resolution of $2 \mathrm{~cm}^{-}$ 1. NAT-NPs, NAT, CTS, and TPP were mixed with $\mathrm{KBr}$ and compressed into pellets. The characteristic peaks of the FT-IR transmission spectra were documented.

Thermal characterizations were also performed by thermogravimetric (TGA) and differential thermal analyses (DTA) by using a TGA Q500 V20 Thermogravimetric Analyzer (TA Instruments, USA). TGA and DTA were carried out at $10^{\circ} \mathrm{C} / \mathrm{min}$ from $0^{\circ} \mathrm{C}$ to $800^{\circ} \mathrm{C}$ at a flow rate of $60 \mathrm{~mL} / \mathrm{min}$ and compared with NAT, CTS, TPP, and NATNPs. 
2.7. In Vitro Drug Release Studies. NAT of the prepared NAT-NPs and free NAT was released in vitro at dissolution media at PBS ( $\mathrm{pH} 7.4)$ at $37^{\circ} \mathrm{C}$. In brief, NAT was dissolved in PBS, and NAT-NPs were dialyzed for 2 days. About $2 \mathrm{~mL}$ of NAT-NPs and NAT solution was immersed in $100 \mathrm{~mL}$ of dialyzed tube of PBS at $37^{\circ} \mathrm{C}$ and $100 \mathrm{rpm}$. About $1 \mathrm{~mL}$ of solution was removed at a predetermined time, and the same amount of buffer was immediately added. To increase NAT solubility, all samples were tested in an environment with and without Tween-80 plus $0.5 \%(\mathrm{v} / \mathrm{v})$. The standard curve of NAT obtained from PBS buffer was used to calculate NAT release. All the experiments were performed in triplicate. The cumulative release rate $(Q \%)$ was calculated as follows:

$$
Q(\%)=\sum_{i=1}^{n=j} C i V i+\frac{C_{n} V}{W} \times D \times 100 \%,
$$

where $C i$ and $\mathrm{C}_{n}$ are the drug concentrations in released medium, $V i$ is the volume of mediator removed, $V$ is the total amount of leaching medium, and $D$ and $W$ are the drug concentrations in the NPs and weight of the NPs, respectively.

\section{In Vitro Antifungal Activity}

3.1. MIC Assays. The influence of the NAT encapsulated NPs and free NAT on antifungal activity was evaluated in terms of MIC50. Sample tests were performed in 96-well plates with serial dilutions of NAT by adding known concentrations of the NP suspensions to liquid Sabouraud medium, as well as free NAT. A series of drug formations of.1, 4.2, 8.45, 16.9, 33.8 , and $67.6 \mu \mathrm{M}$ NAT was made in the medium. Some wells served as the growth control and sterility check. Cell suspensions of $C$. albicans were prepared in DMEM and adjusted to yield a final inoculum concentration of $1.0-5.0 \times 10^{3} \mathrm{CFU} /$ $\mathrm{mL}$. The fungal suspension $(100 \mu \mathrm{L})$ was added to each well, resulting in the desired final drug concentration and inoculum size. The microplates were incubated at $35^{\circ} \mathrm{C}$ for $24 \mathrm{~h}$. Organism control and medium control were performed simultaneously to check growth inhibiting activity, organisms, and sterility of broth medium.

3.2. Inhibition Diameter Diffusion Experiment. The paper diffusion method was used to measure bacteriostatic diameter. A sterile cotton swab was dipped in the bacterial liquid, rotated, and squeezed inside the tube wall to remove the excess bacterial liquid and coated on the entire agar plate surface for three times, rotating the plate each time. Finally, a circle was applied along the edge of the plate. The surface dish cover was placed at room temperature for 3-5 min to make the surface slightly dry. Sterile forceps were used to paste the drug-containing paper flat on the surface of the plate, and the plate was placed in a $35^{\circ} \mathrm{C}$ incubator for 24 and $48 \mathrm{~h}$ within $15 \mathrm{~min}$ after being pasted. The bacteriostatic band was used to measure the bacteriostatic circle. The first group included NAT-NPs, free NAT, and PBS, whereas the second group included NAT-NPs, free NAT, and blank-NPs.
TABLE 1: The results of size, zeta potential, and PDI of different volume ratios (NAT: carrier).

\begin{tabular}{lccc}
\hline $\begin{array}{c}\text { N/C } \\
(\mathrm{v} / \mathrm{v})\end{array}$ & $\begin{array}{c}\text { Particle size } \\
(\mathrm{nm})\end{array}$ & $\begin{array}{c}\text { Polydispersity } \\
\text { index }\end{array}$ & $\begin{array}{c}\text { Zeta potential } \\
(\mathrm{mV})\end{array}$ \\
\hline $1: 20$ & $444.7 \pm 2.4$ & 0.360 & +22.6 \\
$1: 12$ & $364.3 \pm 6.8$ & 0.280 & +35.5 \\
$2: 15$ & $514.8 \pm 7.5$ & 0.246 & +29.0 \\
$1: 6$ & $476.3 \pm 51.4$ & 0.405 & +23.5 \\
$1: 4$ & $725.3 \pm 32.0$ & 0.523 & +21.0 \\
$1: 3$ & $787.1 \pm 9.5$ & 0.324 & +10.9 \\
\hline
\end{tabular}

TABLE 2: The results of encapsulation efficiency and drug loading (mean \pm SD, $n=3, \%$ ).

\begin{tabular}{lcc}
\hline $\mathrm{N} / \mathrm{C}(\mathrm{v} / \mathrm{v})$ & DL $(\%)$ & EE $(\%)$ \\
\hline $1: 20$ & $4.37 \pm 0.06$ & $91.30 \pm 1.40$ \\
$1: 12$ & $7.19 \pm 0.02$ & $92.94 \pm 0.21$ \\
\hline
\end{tabular}

3.3. Statistical Analysis. Data were analyzed with Statistical Package for the Social Sciences version 16.0 software (SPSS Inc., Chicago, IL, USA). The experiments were carried out in triplicates, and values are expressed as the mean \pm standard deviation (SD). Student's $t$-test was conducted to determine significance. Statistical significance was considered at a probability level of $p<0.05$.

\section{Results and Discussion}

4.1. Prepare and Characterization of NAT-NPs. The particle size is an important physical property of NPs that directly affects cellular uptake and biodistribution. Within a certain range, the smaller the particle size is, the better the penetration and absorption of the drug. A strong positive charge can penetrate both the cornea and the conjunctiva due to the positive surface charge and ability to enhance the passive diffusion of the drugs [34]. Therefore, the size and zeta potential of NAT-NPs were our primary concerns. The average diameter and PDI of the NAT-NPs prepared by the ion crosslinking method are shown in Figure 2 and Table 1. Given the different volume ratios of NAT/carrier, particle size and PDI were significantly different. In general, the particle size gradually increased with the increase in the NATto-carrier volume ratio. Considering particle size, PDI, and zeta potential, NAT-NPs were most appropriate when the ratios of NAT-to-carrier volume were $1: 12$ and $1: 20$.

The EE and DL of NAT-NPs are shown in Table 2. NATNPs had high drug encapsulation, which possibly led to the drug's good therapeutic effect. Compared with the results of $1: 20$, the volume at $1: 12$ had higher EE. Thus, the volume at 1:12 was obtained for the following experiment. TEM images were also used to identify the structure of NAT-NPs and predict roughly spherical and subspherical particles (Figure 3(b)). 


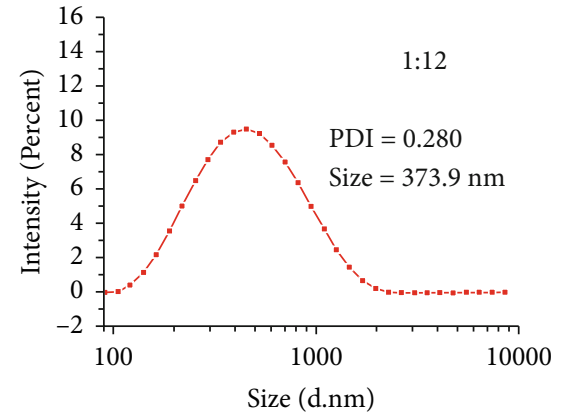

(a)

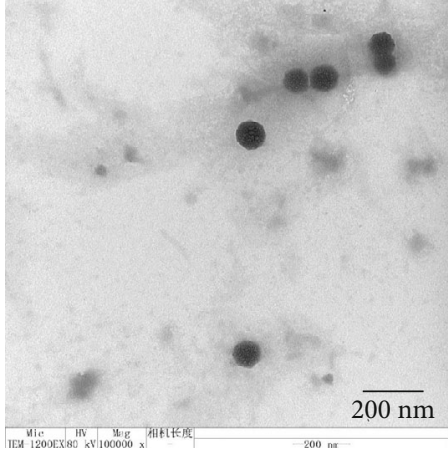

(b)

FIgURE 3: The size distribution, PDI (a), and the morphology using TEM (b) of NAT-NPs (NAT/carrier volume ratio of $1: 12$ ).

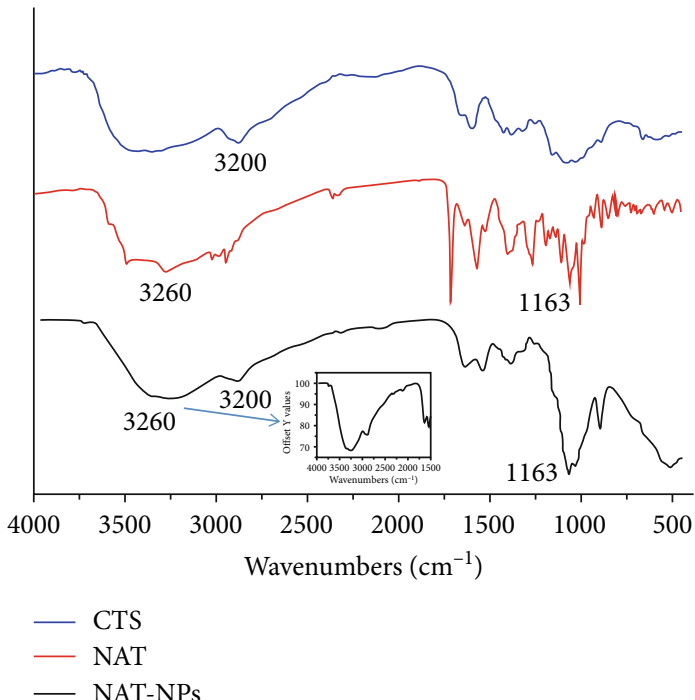

(a)

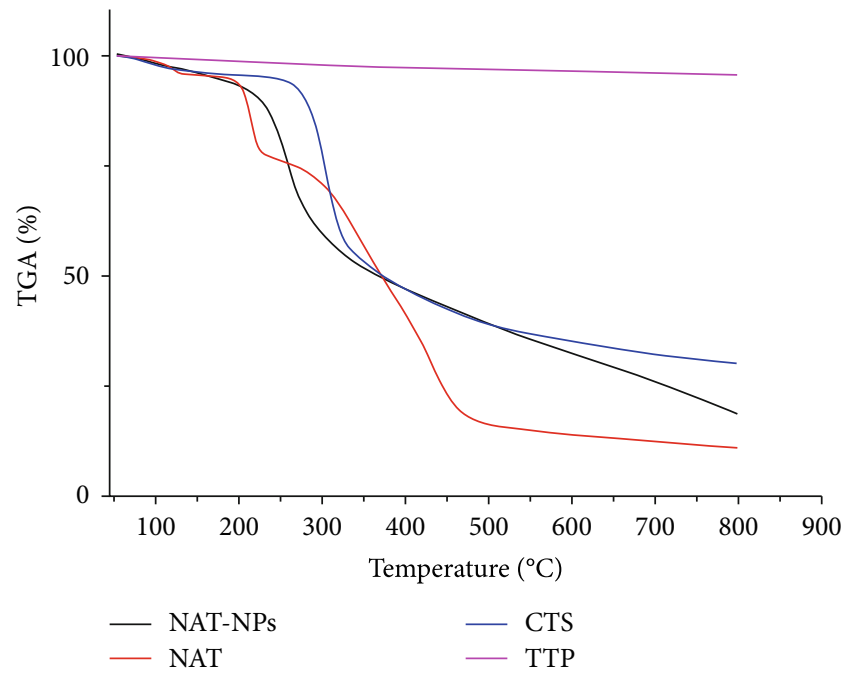

(b)

Figure 4: The FT-IR spectra of CTS, NAT, and NAT-NPs (a). TGA of CTS, TPP, NAT, and NAT-NPs (b).

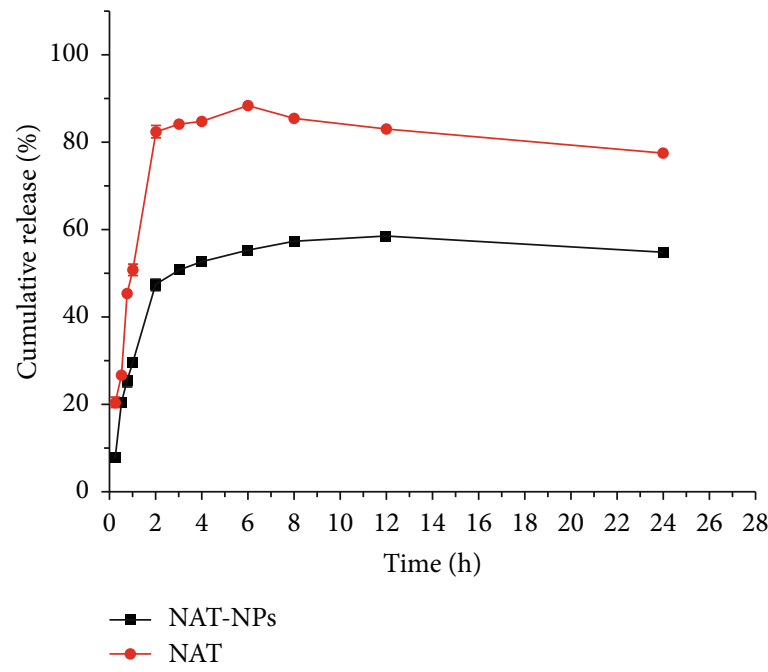

(a)

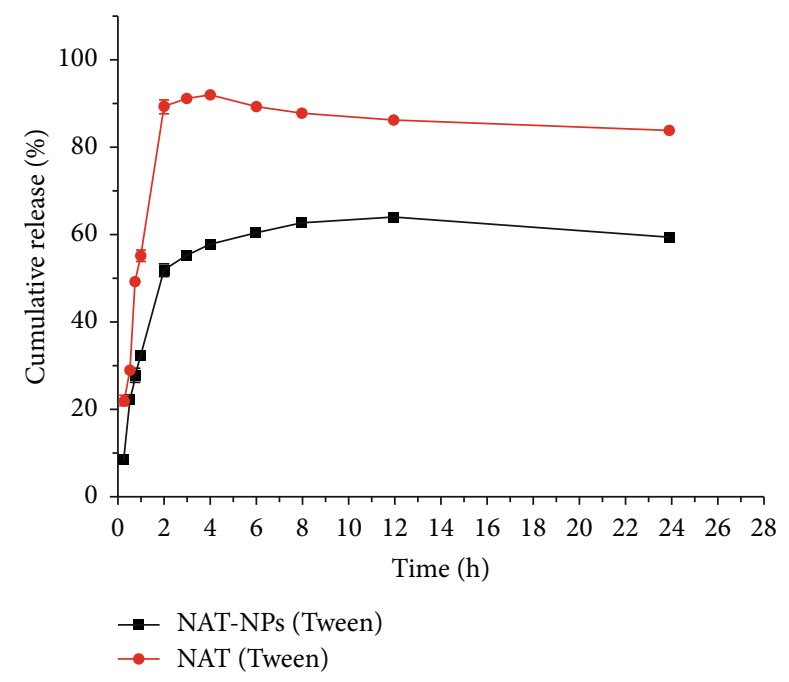

(b)

FIGURE 5: In Vitro release profiles of the optimized NPs (N/C $1: 12, \mathrm{v} / \mathrm{v})$ and NTM with and without $0.5 \%$ Tween-80 (v/v). 


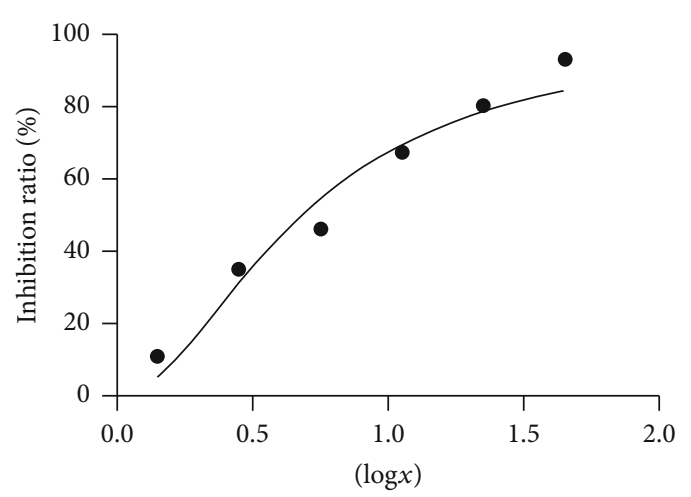

(a)

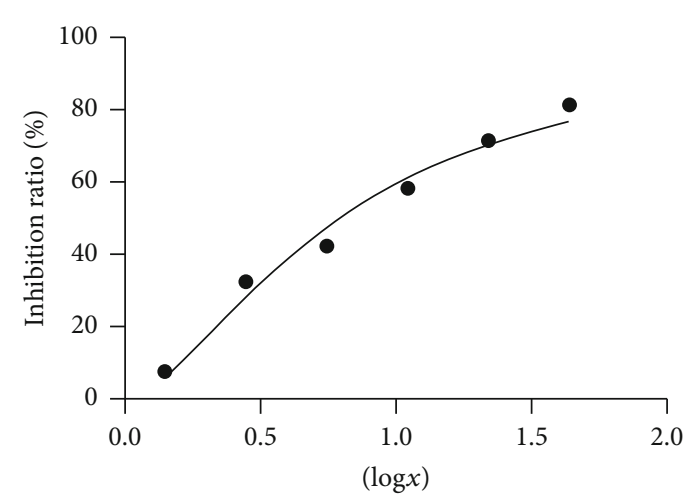

(b)

FIgURE 6: The results IC50 of NAT-NPs (a) and NAT (b) (mean $\pm \mathrm{SD}, n=3, \%$ ).

4.2. Fourier Transform Infrared Spectroscopy (FTIR) and TGA Analysis. FTIR was conducted to identify the structural composition of a substance or determine its chemical group by testing the characteristics of the molecular structure diagram. The FTIR spectra of CTS, NAT, and NAT-NPs are shown in Figure 4(a). The characteristic absorption bands of CTS appeared at 3500 and $3200 \mathrm{~cm}^{-1}$, which were assigned to the $-\mathrm{OH}$ stretching and $-\mathrm{NH}$ bending vibration, respectively. The characteristic absorption bands of NAT appeared at $3260(-\mathrm{NH}), 1640$ (C=C), 1163 (C-CO-C), and 1000 (-C$\mathrm{N}) \mathrm{cm}^{-1}$. For the NAT-NP spectrum, features were observed in bands of $3260(-\mathrm{NH}), 3200(-\mathrm{NH})$, and 1163 (C-CO-C) $\mathrm{cm}^{-1}$ compared with CTS and NAT. These findings suggested an interaction between NAT and the nanocarrier and its successful encapsulation.

TGA and DTA analyses were carried out to determine sample stability and loss of weight in diverse temperatures to measure the physical and chemical changes of the NPs as a function of temperature. In Figure 4(b), the first event, observed from $50^{\circ} \mathrm{C}$ to $140^{\circ} \mathrm{C}(\Delta m=3.78 \%)$, should be linked to the volatilization of water molecules adsorbed on the NAT-NPs surface. NAT-NPs showed intensive weight loss $(\Delta m=43.7 \%)$, which was attributed to the decomposition of the polymer starting from $240^{\circ} \mathrm{C}$ to $360^{\circ} \mathrm{C}$. For CTS and NAT, weight loss appeared in the TG response from $260^{\circ} \mathrm{C}$ to $360^{\circ} \mathrm{C}(\Delta m=43.9 \%)$ and in the TG response from $200^{\circ} \mathrm{C}$ to $520^{\circ} \mathrm{C}(\Delta m=80.2 \%)$, respectively. These TGA data showed some increase in thermal stability of NAT-NPs compared with NAT.

4.3. In Vitro Drug Release. The in vitro drug release of NATNPs and NAT in the simulated ocular circumstances $\left(37^{\circ} \mathrm{C}\right.$, $\mathrm{pH}$ 7.4) was studied (Figure 5). A control group with $0.5 \%$ Tween-80 (v/v) was also established due to the poor water solubility of NAT. The NPs showed a two-step release pattern: one initial burst release within $2 \mathrm{~h}$, followed by a second slow-release phase. The cumulative release of NAT freed from the NAT-NPs was $52.15 \%$ and $47.5 \%$ within $2 \mathrm{~h}$ with and without Tween-80, respectively. Some drug was adsorbed on the surface of NAT-NPs during the preparation, which explained the burst release within the first $2 \mathrm{~h}$. More importantly, an initial burst release is beneficial in terms of
TABLE 3: The results IC50 of NAT-NPs and NAT (mean \pm SD, $n=3, \%)$.

\begin{tabular}{lcc}
\hline Antifungal agent & IC50 $(\mu \mathrm{g} / \mathrm{mL})$ & $\begin{array}{c}95 \% \text { confidence interval } \\
(\mu \mathrm{g} / \mathrm{mL})\end{array}$ \\
\hline NAT-NPs & 4.82 & $4.10-5.65$ \\
NAT & 6.17 & $5.85-7.86$ \\
\hline
\end{tabular}

antifungal activity as it helps achieve the therapeutic concentration of drug in a minimal period followed by constant release to maintain the sustained and controlled release of the drug. The cumulative releases of NAT were $64.32 \%$ and $58.54 \%$ within $12 \mathrm{~h}$ with and without Tween-80, respectively. There was no significant difference in NAT-NPs release between the two environments, but the NAT-NPs showed a stronger sustained release effect than free NAT.

4.4. In Vitro Antifungal Activity of NPs. To assess the antifungal activity of NAT-NPs, the IC50 was determined (Figure 6 and Table 3). The IC50 of NAT-NPs showed stronger antifungal activity than free NAT against C. albicans. To further quantify the antifungal effect of NAT-NPs, we conducted an inhibition zone experiment. The zones of inhibition were defined as the maximum distances between the test disk and the fungal growth edge (recorded in millimeters). The zones of inhibition for NAT-NPs ( $24 \mathrm{~h}$ and $48 \mathrm{~h}$ ) are shown in Figure 7(a-b) and Table 4, respectively. Whether at 24 or $48 \mathrm{~h}$, the antifungal effect of NAT-NPs was much higher than that of NAT, which was consistent with the result of IC50. As expected, the zone of inhibition gradually decreased over time. We speculated that the reason for improving the antifungal effect of NAT-NPs was the increase in water solubility (increased bioavailability) of NAT and the antifungal effect of blank-NPs (CTS) themselves. To further verify the effect of blank-NPs on C. albicans, we compared the antifungal ability of NAT-NPs, blank-NPs, and NAT through the inhibition zone experiment Figure 7(c-d) and Table 5. CTS-NPs also presented considerable antifungal effects and were stronger than free NAT. Notably, the antifungal effect of NAT-NPs was far stronger than that of NAT and CTS. A synergistic effect may exist between NAT and CTS. These results may provide useful information for the facile application of NAT-NPs. 


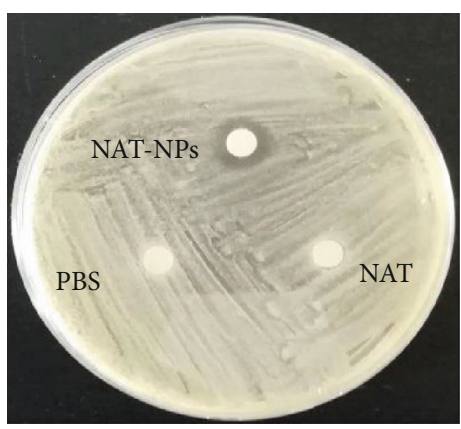

$24 \mathrm{~h}$

(a)

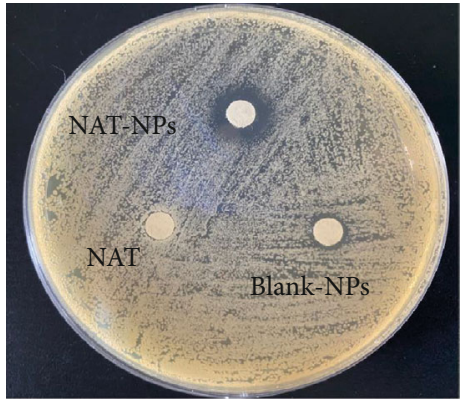

$24 \mathrm{~h}$

(c)

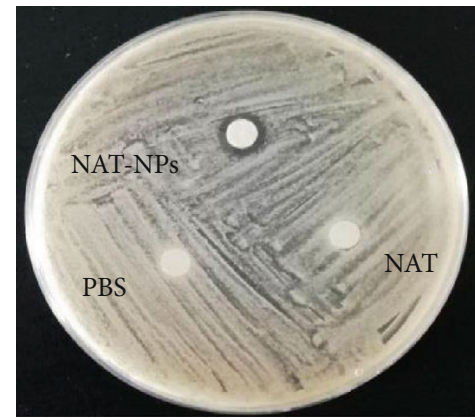

$48 \mathrm{~h}$

(b)

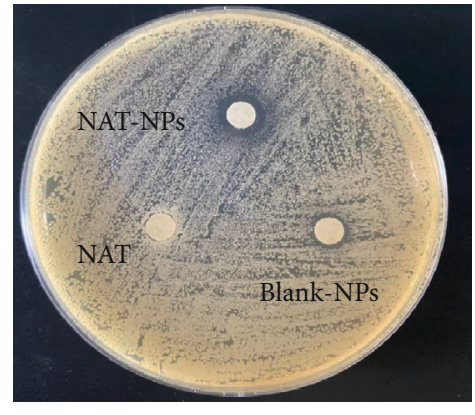

$48 \mathrm{~h}$

(d)

Figure 7: The zone of inhibition of NAT-NPs, NAT, and PBS within $24 \mathrm{~h}(\mathrm{a})$ and $48 \mathrm{~h}(\mathrm{~b})$. The zone of inhibition of NAT-NPs, NAT, and blank-NPs within $24 \mathrm{~h} \mathrm{(c)} \mathrm{and} 48 \mathrm{~h}(\mathrm{~d})$.

TABLE 4: The results of the inhibitory zone diameter $(\mathrm{mm})$ of NATNPs, NAT, and PBS (mean $\pm \mathrm{SD}, n=3, \mathrm{~mm}$ ).

\begin{tabular}{lcc}
\hline Inhibitory zone diameter & $24 \mathrm{~h}(\mathrm{~mm})$ & $48 \mathrm{~h}(\mathrm{~mm})$ \\
\hline NAT-NPs & $10.57 \pm 0.13$ & $9.15 \pm 0.08$ \\
NAT & $6.20 \pm 0.12$ & $6.09 \pm 0.03$ \\
PBS & 6.0 & 6.0 \\
\hline
\end{tabular}

TABLE 5: The results of the inhibitory zone diameter ( $\mathrm{mm})$ of NATNPs, NAT, and blank-NPs (mean $\pm \mathrm{SD}, n=3, \%$ ).

\begin{tabular}{lcc}
\hline Inhibitory zone diameter & $24 \mathrm{~h}(\mathrm{~mm})$ & $48 \mathrm{~h}(\mathrm{~mm})$ \\
\hline NAT-NPs & $11.32 \pm 0.18$ & $9.96 \pm 0.05$ \\
NAT & $6.25 \pm 0.03$ & $6.15 \pm 0.04$ \\
Blank-NPs & $8.08 \pm 0.17$ & $7.25 \pm 0.08$ \\
\hline
\end{tabular}

\section{Conclusions}

In this study, NAT-NPs exhibited good physicochemical properties and proved that NAT-NPs had a strong antifungal effect on C. albicans. The reason why NAT-NP had a stronger antifungal effect than NAT was related to the antifungal properties of CTS itself. CTS enhances NAT in the antifungal direction, making CTS an excellent carrier of NAT. Therefore, NAT-NPs is expected to be effective in clinical fungal keratitis due to its potent ability to inhibit fungi. Although the NPs formed in this paper demonstrated the slow-release effect of NAT-NPs and strong antifungal effects, further studies should be conducted to enable NAT-NPs to be applied earlier in clinical patients with fungal keratitis.

\section{Data Availability}

All the data of the study can be obtain by contacting with the corresponding author.

\section{Conflicts of Interest}

The author(s) declare(s) that they have no conflicts of interest.

\section{Acknowledgments}

The authors are indebted to the financial support from the National Natural Science Foundation of China (Nos. 81774125, 81973671), the Natural Science Foundation of Shandong Province (No. ZR2019BB071), and the Major Innovation and Technology Engineering Project of Shandong Province (No. 2019JZZY011106).

\section{References}

[1] D. He, J. Hao, B. Zhang et al., "Pathogenic spectrum of fungal keratitis and specific identification of Fusarium solani," 
Investigative Ophthalmology \& Visual Science, vol. 52, no. 5, pp. 2804-2808, 2011.

[2] P. K. Shukla, M. Kumar, and G. B. Keshava, "Mycotic keratitis: an overview of diagnosis and therapy," Mycoses, vol. 51, no. 3, pp. 183-199, 2008.

[3] P. A. Thomas, "Fungal infections of the cornea," Eye (London, England), vol. 17, no. 8, pp. 852-862, 2003.

[4] T. Sirikul, T. Prabriputaloong, A. Smathivat, R. S. Chuck, and A. Vongthongsri, "Predisposing factors and etiologic diagnosis of ulcerative keratitis," Cornea, vol. 27, no. 3, pp. 283-287, 2008.

[5] F. Mellado, T. Rojas, and C. Cumsille, "Fungal keratitis: review of diagnosis and treatment," Arquivos Brasileiros de Oftalmologia, vol. 76, no. 1, pp. 52-56, 2013.

[6] M. Srinivasan, "Fungal keratitis," Current Opinion in Ophthalmology, vol. 15, no. 4, pp. 321-327, 2004.

[7] N. V. FlorCruz, I. V. Peczon, and J. R. Evans, "Medical interventions for fungal keratitis," Cochrane Database of Systematic Reviews, vol. 2, article CD004241, 2012.

[8] N. V. FlorCruz and I. V. Peczon, "Medical interventions for fungal keratitis," Cochrane Database of Systematic Reviews, vol. 1, article CD004241, 2008.

[9] Y. F. Li, J. Jin, Q. Guo, Y. M. Ha, and Q. P. Li, "Complexation of synthetic CDM-AM copolymer with natamycin and carbendazim to improve solubility and fungicidal activity," Carbohydrate Polymers, vol. 125, pp. 288-300, 2015.

[10] U. B. Kompella, A. C. Amrite, R. Pacha Ravi, and S. A. Durazo, "Nanomedicines for back of the eye drug delivery, gene delivery, and imaging," Progress in Retinal and Eye Research, vol. 36, pp. 172-198, 2013.

[11] D. Gulsen and A. Chauhan, "Ophthalmic drug delivery through contact lenses," Investigative Ophthalmology \& Visual Science, vol. 45, no. 7, pp. 2342-2347, 2004.

[12] J. Kim, A. Conway, and A. Chauhan, "Extended delivery of ophthalmic drugs by silicone hydrogel contact lenses," Biomaterials, vol. 29, no. 14, pp. 2259-2269, 2008.

[13] T. Bourcier, A. Sauer, A. Dory, J. Denis, and M. Sabou, "Fungal keratitis," Journal Français d'Ophtalmologie, vol. 40, no. 9, pp. e307-e313, 2017.

[14] G. G. Müller, N. Kara-José, and R. S. . Castro, "Antifúngicos em infecções oculares: drogas e vias de administração," Revista Brasileira de Oftalmologia, vol. 72, no. 2, pp. 132141, 2013.

[15] P. W. Morrison and V. V. Khutoryanskiy, "Advances in ophthalmic drug delivery," Therapeutic Delivery, vol. 5, no. 12, pp. 1297-1315, 2014.

[16] D. R. Janagam, L. Wu, and T. L. Lowe, "Nanoparticles for drug delivery to the anterior segment of the eye," Advanced Drug Delivery Reviews, vol. 122, pp. 31-64, 2017.

[17] R. Herrero-Vanrell, M. Vicario de la Torre, V. Andrés-Guerrero, D. Barbosa-Alfaro, I. T. Molina-Martínez, and I. BravoOsuna, "Nano and microtechnologies for ophthalmic administration, an overview," Journal of Drug Delivery Science and Technology, vol. 23, no. 2, pp. 75-102, 2013.

[18] A. Urtti, "Challenges and obstacles of ocular pharmacokinetics and drug delivery," Advanced Drug Delivery Reviews, vol. 58, no. 11, pp. 1131-1135, 2006.

[19] S. L. Fialho and A. da Silva-Cunha, "New vehicle based on a microemulsion for topical ocular administration of dexamethasone," Clinical \& Experimental Ophthalmology, vol. 32, no. 6, pp. 626-632, 2004.
[20] A. M. de Campos, A. Sánchez, R. Gref, P. Calvo, and M. J. Alonso, "The effect of a PEG versus a chitosan coating on the interaction of drug colloidal carriers with the ocular mucosa," European Journal of Pharmaceutical Sciences, vol. 20, no. 1, pp. 73-81, 2003.

[21] M. Kassem, A. Abdelrahman, M. Ghorab, M. Ahmed, and R. Khalil, "Nanosuspension as an ophthalmic delivery system for certain glucocorticoid drugs," International Journal of Pharmaceutics, vol. 340, no. 1-2, pp. 126-133, 2007.

[22] D. Owensiii and N. Peppas, "Opsonization, biodistribution, and pharmacokinetics of polymeric nanoparticles," International Journal of Pharmaceutics, vol. 307, no. 1, pp. 93-102, 2006.

[23] A. A. Badawi, H. M. El-Laithy, R. K. El Qidra, H. El Mofty, and M. El dally, "Chitosan based nanocarriers for indomethacin ocular delivery," Archives of Pharmacal Research, vol. 31, no. 8, pp. 1040-1049, 2008.

[24] R. C. Nagarwal, R. Kumar, and J. K. Pandit, "Chitosan coated sodium alginate-chitosan nanoparticles loaded with 5-FU for ocular delivery: In vitro characterization and in vivo study in rabbit eye," European Journal of Pharmaceutical Sciences, vol. 47, no. 4, pp. 678-685, 2012.

[25] M. A. Kalam, "The potential application of hyaluronic acid coated chitosan nanoparticles in ocular delivery of dexamethasone," International Journal of Biological Macromolecules, vol. 89, pp. 559-568, 2016.

[26] R. Sharma, M. Ahuja, and H. Kaur, "Thiolated pectin nanoparticles: Preparation, characterization and ex vivo corneal permeation study," Carbohydrate Polymers, vol. 87, no. 2, pp. 1606-1610, 2012.

[27] H. Vaghari, H. Jafarizadeh-Malmiri, A. Berenjian, and N. Anarjan, "Recent advances in application of chitosan in fuel cells," Sustainable Chemical Processes, vol. 1, no. 1, p. 16, 2013.

[28] S. A. Agnihotri, N. N. Mallikarjuna, and T. M. Aminabhavi, "Recent advances on chitosan-based micro- and nanoparticles in drug delivery," Journal of Controlled Release, vol. 100, no. 1, pp. 5-28, 2004.

[29] J. Kumirska, M. X. Weinhold, J. Thöming, and P. Stepnowski, "Biomedical activity of chitin/chitosan based materials-influence of physicochemical properties apart from molecular weight and degree of $\mathrm{N}$-acetylation," Polymers, vol. 3 , no. 4 , pp. 1875-1901, 2011.

[30] X. Wang, S. Wang, and Y. Zhang, "Advance of the application of nano-controlled release system in ophthalmic drug delivery," Drug Delivery, vol. 23, no. 8, pp. 2897-2901, 2016.

[31] O. M. Sharaf, M. S. Al-Gamal, G. A. Ibrahim et al., "Evaluation and characterization of some protective culture metabolites in free and nano-chitosan-loaded forms against common contaminants of Egyptian cheese," Carbohydrate Polymers, vol. 223, article 115094, 2019.

[32] R. S. Bhatta, H. Chandasana, Y. S. Chhonker et al., "Mucoadhesive nanoparticles for prolonged ocular delivery of natamycin: in vitro and pharmacokinetics studies," International Journal of Pharmaceutics, vol. 432, no. 1-2, pp. 105-112, 2012.

[33] F. Sonvico, A. Cagnani, A. Rossi et al., "Formation of selforganized nanoparticles by lecithin/chitosan ionic interaction," International Journal of Pharmaceutics, vol. 324, no. 1, pp. 67-73, 2006.

[34] A. R. N. Pontillo and A. Detsi, "Nanoparticles for ocular drug delivery: modified and non-modified chitosan as a promising biocompatible carrier," Nanomedicine (London, England), vol. 14, no. 14, pp. 1889-1909, 2019. 